\title{
Transposable elements in cancer as a by-product of stress-induced evolvability
}

\author{
Tobias Mourier $^{1}{ }^{*}$, Lars P. Nielsen $^{2}$, Anders J. Hansen ${ }^{1}$ and Eske Willerslev ${ }^{1}$ \\ ${ }^{1}$ Natural History Museum of Denmark, Centre for GeoGenetics, University of Copenhagen, Copenhagen, Denmark \\ 2 Department of Virology and the Danish National Biobank, Statens Serum Institut, Copenhagen, Denmark
}

\section{Edited by:}

Cedric Feschotte, University of Utah

School of Medicine, USA

\section{Reviewed by:}

Bernardo Lemos, Harvard University, USA

Edward Chuong, University of Utah School of Medicine, USA

*Correspondence:

Tobias Mourier, Natural History Museum of Denmark, Centre for GeoGenetics, University of Copenhagen, Oester Voldgade 5-7, DK-1350, Copenhagen, Denmark e-mail:tmourier@snm.ku.dk
Transposable elements (TEs) are ubiquitous in eukaryotic genomes. Barbara McClintock's famous notion of TEs acting as controlling elements modifying the genetic response of an organism upon exposure to stressful environments has since been solidly supported in a series of model organisms. This requires the TE activity response to possess an element of specificity and be targeted toward certain parts of the genome. We propose that a similar TE response is present in human cells, and that this stress response may drive the onset of human cancers. As such, TE-driven cancers may be viewed as an evolutionary by-product of organisms' abilities to genetically adapt to environmental stress.

Keywords: transposable elements, stress response, evolution, evolvability, cancer

\section{TRANSPOSABLE ELEMENTS AND GENOME EVOLUTION}

Transposable elements (TEs) and their remnants constitute between half and two thirds of the human genome (Lander et al., 2001; de Koning et al., 2011), and are with few exceptions (Arkhipova and Meselson, 2000; Gardner et al., 2002) found in all sequenced eukaryotic genomes. TEs are divided according to the presence or absence of an RNA intermediate in their replication cycle (elements referred to as retroelements or DNA transposons, respectively). Retroelements are further divided into elements with or without long terminal repeat (LTR) sequences (Kazazian, 2004). The activity of DNA transposons in the human lineage ceased around 40 million years ago and now constitute $3 \%$ of the extant human genome (Lander et al., 2001; Pace and Feschotte, 2007). No LTR retroelements are considered to be actively spreading in the human genome, although the endogenous retrovirus, HERV-K is polymorphic among human populations (Belshaw et al., 2005) and capable of forming viral particles (Boller et al., 2008). In total, endogenous retroviruses constitute around $8 \%$ of the human genome. Three non-LTR retroelements are actively spreading in the human genome; the autonomous L1 LINEs and the two non-autonomous SINEs, Alu and SVA. Full-length L1 LINE elements are around 6 kilo base pairs (kbp) in size and contain two open reading frames encoding the enzymes required for transposition (Ostertag and Kazazian, 2001). More than half a million L1 sequences constitute approximately $17 \%$ of the human genome, although only around 150 elements are full-length (Penzkofer et al., 2005) and presumably even fewer - perhaps only a handful - actively transpose (Brouha et al., 2003). Alu and SVA elements are short ( $~ 300 \mathrm{bp}$ and typically $<1 \mathrm{kbp}$, respectively) and do not encode any proteins (Batzer and Deininger, 2002; Wang et al., 2005). Rather, these elements rely on the enzymatic activity provided by $\mathrm{L} 1$ elements [established for Alu and presumed for SVA (Dewannieux et al., 2003; Ostertag et al., 2003)].
The human genome harbors more than 1.5 million Alu copies and around 500 SVA copies, covering $11 \%$ of the total genomic sequence.

\section{CELLULAR TE REPRESSION}

The activity of TE can be repressed in several ways and at different stages of activity. Most notably, DNA or histone methylation abolishes transcription of TE sequences (Slotkin and Martienssen, 2007). The production of double-stranded RNAs (dsRNAs) from TE sequences may result in the production of small-interference RNAs (siRNAs) targeting transcribed TE sequences for degradation (Ahlquist, 2002; Yang and Kazazian, 2006), or Piwi interacting RNAs that guides de novo methylation during early development (Aravin et al., 2007; Carmell et al., 2007; Kuramochi-Miyagawa et al., 2008). Generation of TE dsRNA sequences may stem from transcription of both strands within specific TE sequences (Yang and Kazazian, 2006; Li et al., 2014). Alternatively, different loci could produce complementary TE RNA strands, as for example from the "passive" transcription of TEs residing in mRNA introns (Mourier, 2011).

L1, Alu, and SVA transcripts may undergo RNA editing through C-to-U deamination by members of the APOBEC3 protein family, inhibiting transposition (Schumann, 2007), and the Trex1 endonuclease metabolizes reverse-transcribed DNA from human L1 sequences and mice LTR elements in human cell cultures (Stetson et al., 2008).

The ORF1 protein from L1 is sequestered in stress granules where it co-localizes with the siRNA-processing RISC complex and closely associates with the putative RNA helicase MOV10 (Goodier et al., 2007, 2012). It is proposed that MOV10 recruits L1 ribonucleoproteins to stress granules, leading to silencing and degradation (Goodier et al., 2012). Interestingly, the MOV10 paralog MOV10L1 binds MILI and MIWI proteins that 
associate with piRNAs, and the knockout of MOV10L1 leads to increased L1 and LTR transcription in mice (Frost et al., 2010).

As previously pointed out, the redundancy between different TE repression mechanisms provides functional strength but also a vulnerability due to interdependence (Carreira et al., 2014). Despite the plethora of suppression mechanisms, TEs are continuing their activity in our genomes as witnessed by the level of TE polymorphisms between humans and chimpanzee (Hedges etal., 2004; Lee etal., 2007), between human individuals (Bennett et al., 2004; Wang et al., 2006; Stewart et al., 2011) and between homologous chromosomes within individuals (Levy et al., 2007; Wang et al., 2008). Importantly, human TE transcription and transposition has been recorded within somatic tissues of individuals (Belancio et al., 2010; Baillie et al., 2011).

\section{TEs AND GENOME EVOLUTION}

If inserted into existing structures, TEs may disrupt genomic functions. However, this so-called insertional mutagenesis is by no means the only way genetic functions can be altered by TE activity. Inserted TE sequences may act as promoters driving transcription of neighboring genes, which is most prominent for LTR elements (Dunn et al., 2003, 2006) but is also observed for hypomethylated LINEs in cancer tissues (Roman-Gomez et al., 2005; Wolff et al., 2010). If inserted within transcribed genetic sequences, L1 elements may repress gene expression by inhibiting transcriptional elongation (Han etal., 2004). A survey of cap-selected human transcripts revealed that $5-15 \%$ of all transcripts from different tissues were initiated within TEs (Faulkner et al., 2009) testifying the impact TE sequences have on the total transcriptome. Ectopic recombination between nonhomologous TEs leading to chromosomal changes has been inferred for all types of human TEs (Hughes and Coffin, 2001; Han etal., 2005; Sen etal., 2006). The reverse transcriptase machinery from L1 elements may occasionally insert exogenous mRNAs, resulting in the formation of processed pseudogenes (Esnault etal., 2000), of which there are around 8000 in the human genome (Zhang etal., 2003). Similarly, if the transcription of L1 elements continues into flanking sequence and genes, these chimerical transcripts may be reverse transcribed and inserted resulting in so-called sequence transduction which is estimated to constitute around $1 \%$ of the human genome (Pickeral et al., 2000). Alu elements residing in untranslated regions and introns often provide splice signals leading to the creation of novel exons (Lin et al., 2008; Keren etal., 2010), and are targets for RNA editing (Paz-Yaacov et al., 2010; Bazak et al., 2014), the level of which have implications for gene regulations (Chen et al., 2008). Furthermore, the transcriptional activity of the murine SINE B2 was shown to act as an insulator for chromatin modification between genomic domains (Lunyak etal., 2007). Notable examples of TE being recruited for genomic functions through evolution include the syncytin genes in placentas, where the envelope proteins from endogenous retroviruses promote the cell fusions between mother and fetus (Haig, 2012; Chuong, 2013), and the enhancer activity of an ancient SINE regulating neural development in mammals
(Bejerano et al., 2006). Accordingly, TEs have contributed significantly to mammalian genome evolution by either creating or deleting elements, changing activity of existing elements, or shuffling genomic regions (Deininger et al., 2003; Kazazian, 2004; Mourier, 2005; Cordaux and Batzer, 2009; Chuong et al., 2013).

\section{CANCER AND EVOLVABILITY}

TE activity is readily reported in cancer cells (Asch et al., 1996; Faulkner et al., 2009; Lamprecht et al., 2010; Romanish et al., 2010; Levin and Moran, 2011; Gualtieri et al., 2013) and is commonly associated with an overall breakdown of cellular TE repression mechanisms, such as methylation (Wilson et al., 2007; Daskalos et al., 2009; Wolff et al., 2010; Xiang et al., 2010; Pavicic et al., 2012). Implicitly, this suggests that increased TE activity is a derived and in essence non-adaptive response in cancer cells. Yet, TE activity during stress may - from an evolutionary perspective - be viewed as a means by which organisms can keep up rates of genetic adaptations to changing conditions. Changing environments stress an organism and cause fixation of favorable genetic changes by natural selection. This again results in genetic adaptation of the species or cells. Here, we argue that cancer related to human $\mathrm{TE}$ activity can be viewed as a byproduct of genome flexibility meant for effectively adapting the cell (Figure 1). And, importantly, that TE activity induced by external stresses should thus be regarded as an evolutionary adaptive mechanism.

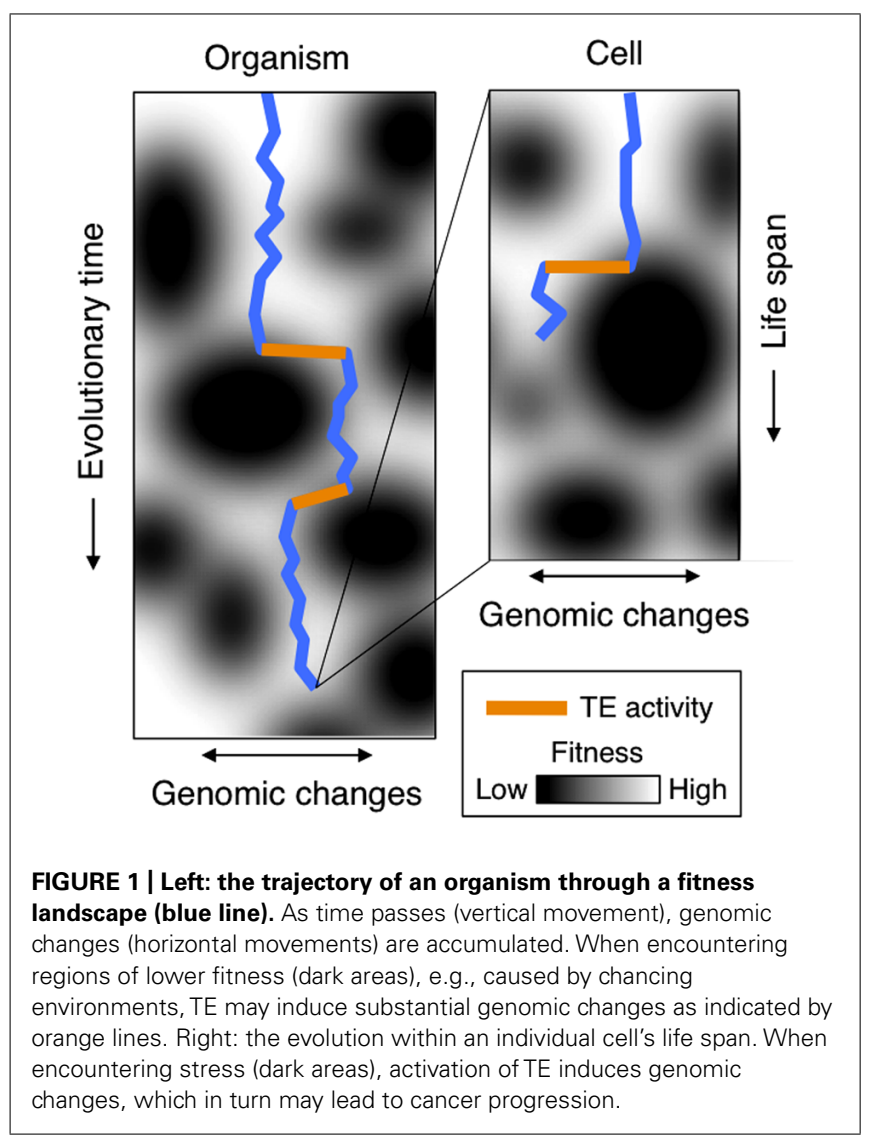


Given the functional diversity of human TEs, a global activation of the entire TE population would provide a crude measure to affect the genotype. Rather, one would imagine that the probability of TEs creating adaptive genotypes increases with specificity (different activity levels for different TEs) and/or targeting (TEs are predominantly inserted in certain regions of the genome). In the following, we briefly review examples of highly targeted TE activity from non-mammalian model organisms, and end by providing observations suggesting that stress-induced TE activity in mammalian cells can be both specific as well as targeted. Hence, environmental stimuli may trigger the formation of genetic changes through TE activity at all stages of cancers, including the very onset.

\section{TES AND EVOLVABILITY}

All living organisms face an apparent paradox. On the one hand, their replication over time should be as exact as possible to maintain proper function, but on the other hand, organisms must evolve for their lineage to survive changing environments. When cells encounter changing environments (whether permanently or temporarily, the latter interchangeably referred to as stress) they may induce genomic changes potentially generating adaptive mutations.

When multiple genes carry out overlapping functions, individual genes are allowed to diverge from their optimum without affecting the phenotypic outcome (Hermisson and Wagner, 2004), allowing an exploration of genotype space with little non-adaptive cost (Masel and Trotter, 2010). Hence, genomes will carry in them a range of mutations (termed cryptic variation) that can be released by the activity of so-called genetic capacitors (Rutherford and Lindquist, 1998; Bergman and Siegal, 2003; Masel and Trotter, 2010). In this context, it is noteworthy that inhibition of the capacitor hsp90 deregulates the activity of TEs (Specchia etal., 2010), suggesting a role for TEs in regulators of evolvability.

Importantly, the ability to generate genetic variability, termed evolvability, does not imply foresight or teleology (Poole et al., 2003). Errors introduced by the replication machinery thus represent one factor contributing to evolvability. Compared to single base pair substitutions, inducing TE activity provides a highly efficient way of generating genetic variability. Although the vast majority of TE insertions are deleterious (Boissinot etal., 2001, 2006), they may occasionally modify existing gene structures or merely alter the expression profiles of genes (both temporally and spatially) in a way that proves adaptive to the host.

Introducing genomic changes when facing environmental stress - as suggested by the TE pioneer Barbara McClintock (McClintock, 1984) - makes intuitive sense for organisms with limited mobility, such as plants and unicellular organisms that are unable to evade suboptimal environments and are forced to adapt to the external changes. Among such organisms it is well established that cellular stresses can lead to highly specific TE activity, often minimizing deleterious insertions and maximizing the potential for adaptive regulatory changes. Below we review a few selected examples of specific TE activity in non-mammalian model organisms.

\section{EXAMPLES OF STRESS-INDUCED TE ACTIVITY IN MODEL ORGANISMS}

Stress-induced TE activity is found across a multitude of organisms (Arnault and Dufournel, 1994; Capy et al., 2000; Morales et al., 2003; Garcia Guerreiro, 2012), and although this may reflect a genome-wide collapse of cellular TE suppression, numerous examples of specifically induced TE activity in response to stress are known. In compact genomes, TE insertions are usually highly targeted toward genomic regions where interference with endogenous functions is minimized. This is seen in fission yeast where LTR elements are preferentially inserted upstream of polymerase II transcribed genes (Bowen et al., 2003; Leem et al., 2008). Upon low oxygen levels, a specific transcription factor induces LTR transposition in fission yeast and in turn, transcription of downstream genes (Sehgal et al., 2007). A similar specific activation is found in budding yeast, where depletion of adenylic nucleotides induces a transcription factor, activating the LTR transcription (Servant et al., 2012). In the tobacco plant, Nicotiana tabacum, the Tnt1 retrotransposon is induced by abiotic stresses (Mhiri et al., 1997) and share transcriptional activation regions with stress-inducible genes (Grandbastien et al., 1997).

The insertion of Ty5 LTR elements in budding yeast is targeted toward heterochromatic regions through the binding between the Ty5 integrase and a heterochromatin component (Gai and Voytas, 1998). Upon exposure to cellular stress factors, integrase phosphorylation decreases which abolishes the targeting and results in insertions near genes, potentially altering their function and activity (Ebina and Levin, 2007). Hence, the result of stress is in this case not restricted to the sheer magnitude of TE activity, but rather on the potential effect TE activity may impose on the genotype. In the above cases, TE activity is a direct, inducible result of stress, and not the indirect by-product of cellular turmoil.

That the genotypic effect of TE activity that penetrates to the phenotype is illustrated in fission yeast, where heat-induced TE activity changes the expression of stress genes downstream to newly inserted TE sequences (Feng et al., 2012), leaving an immediate effect on gene functionality. Some fixed TE insertions fit well with being triggered by stress, and eventually conferring the host with a modified - and adaptive - response toward the stressor. Such examples include the soybean, Glycine max in which the disruption of the gmphyA2 gene by a TE insertion is associated with high latitudes (Kanazawa et al., 2009), the mosquito, Culex pipiens where the disruption of the $c p m 1$ receptor gene by a TE insertion confers resistance to a specific toxin (Darboux et al., 2007), and Drosophila where a TE-mediated gene truncation increases pesticide resistance (Aminetzach et al., 2005).

\section{SPECIFICITY AND TARGETING OF MAMMALIAN TE ACTIVITY}

If mammalian stress-induced TE activity reflects the evolvability observed in other model organisms, TE activity is expected to be non-random. First, insertions should be targeted so that certain genomic regions are preferential targets for TE insertions. Second, given the functional diversity of mammalian TEs, the response should be specific, so that different TEs are activated by different environmental stress factors. In the following, we review findings 
suggesting that mammalian TE-activity can be both specific and targeted.

During heat shock, mammalian cells up-regulate a specific set of genes encoding heat shock proteins while repressing a large repertoire of otherwise constitutively expressed genes (Richter et al., 2010). In mammals, the polymerase III (pol III) transcribed SINEs are up-regulated during heat shock and enter the complexes that target promoters of repressed genes (Ponicsan et al., 2010). Intriguingly, in humans and mouse two different and unrelated SINEs [the human Alu is derived from a 7SL RNA (Batzer and Deininger, 2002), the mouse B2 from a tRNA (Daniels and Deininger, 1985)] are part of this heat shock response (Allen et al., 2004; Mariner et al., 2008). Hence, different SINEs have been recruited for this task during mammalian evolution, suggesting that increased transcription during heat shock is a common feature of these SINEs. Importantly, heat shock activation is specific to SINEs as other pol III transcribed genes are unaffected (Liu et al., 1995), suggesting that increased SINE expression is not simply a result of a general increase in pol III activity.

Hunter and colleagues recently reported the silencing of TE sequences through histone methylation in the brains of stressed rats (Hunter et al., 2012). Interestingly, this revealed a surprising differentiation between TE types and brain regions, and most strikingly that histone methylation did not target L1 sequences. As transgenic L1s are in fact actively transposing in rodent hippocampus (Muotri et al., 2005; Kuwabara et al., 2009), there is no reason to suspect that $\mathrm{L} 1$ should be less active than other rodent TEs in this part of the brain. This differential silencing is consistent with methylation not acting as a global response to an overall increase in TE activity. Although the observed differences between TE activity levels may reflect the diversity by which different TEs are normally repressed - so that specific activation is a result of specific de-repression - it shows that an external stimulus may elicit a specific TE response rather than being restricted to a global elevation of TE activity. A recent example comes from the discovery of hypomethylation of specific human TE subfamilies in a tissue-specific manner, resulting in the gain of enhancer marks, which strongly correlated with the regulation of nearby genes (Xie et al., 2013).

One element of TE targeting comes directly from the fact that integrations are restricted to open chromatin regions. This is apparent in mammalian brains where somatic LINE insertions are enriched in the vicinity of neuronal genes (Muotri et al., 2005; Baillie et al., 2011). The finding that different environmental factors induce L1 transposition through different bHLH/PAS proteins opens the possibility of differentiated targeting of L1 insertions during different types of stresses (Ishizaka et al., 2012), and experimental findings hint that TE insertions are targeted beyond the apparent mechanistic necessity of open chromatin. Howard and colleagues created transgenic mice with a functional loss of DNA methyltransferase activity, which results in genomic hypomethylation and development of thymic lymphomas (Howard et al., 2008). In a sample of 16 transgenic mice, 7 were found to have independent IAP insertions into the introns of the Notch gene (Howard et al., 2008). Similarly, Wimmer et al. reported a genomic hotspot for TE insertions in the neurofibromin 1, NF1 gene (Wimmer et al., 2011), and more than a handful of human genomic loci are known for which multiple, independent TE insertions are reported (Chen et al., 2005; Hancks and Kazazian, 2012). Finally, it should be noted that wide variations in the rates between mobilized TEs are observed among different human cancers (Lee et al., 2012).

Interestingly, apparent TE targeting can be observed over evolutionary times, as a study on the contribution of TE sequences to human mRNA untranslated regions found genes responding to stress and external stimuli to harbor more TE sequence than other gene classes (van de Lagemaat et al., 2003). There is obviously a strong ascertainment bias in the above observations as only certain phenotypic outcomes are considered and that highly deleterious insertions are immediately pruned by natural selection. Yet, this nevertheless suggests that the genomic distribution of human TE insertions is far from random.

\section{CONCLUDING REMARKS}

TEs typically contain several functional components that can be moved around the genome and inserted into novel genetic contexts. As different environmental stimuli may elicit differential activity of TE classes, and as insertions can be highly non-random, it is apparent that TEs provide a highly efficient mechanism for evolvability.

In line with previous speculations (Hauptmann and Schmitt, 2006), we have suggested that stress-induced TE activity driving human cancers is a reminiscent of TE-inducible evolvability (Figure 1). However, we here address the different evolution and ecology of eukaryotic cells in single and multicellular organisms, which is essential for this discussion.

We have highlighted examples of mammalian stress-induced TE activity being both targeted and specific. Importantly, TE activity may not necessarily be limited to transposition but could consist of transcriptional activity only. Whether the ability of stress-inducible TE activity serves as an adaptive host response within our cells or it reflects an inherent ability of TEs of being activated upon changing environments is unknown. Perhaps, the innate properties that have allowed the exaptation of TEs in cellular stress responses (such as the heat shock response, above) are the very same properties that underlie the ability to induce evolvability. This way, these properties would be selected for because of the former (heat shock) without the latter (evolvability) necessarily being adaptive. In organisms with a sequestered germ line, somatic TE insertions will not be passed on to future generations. Yet it is possible that somatic cells and tissues modify their hard-coded genetic information during an individual's life span. The notion of the mammalian genome as a stable and inert code may largely result from our experimental inability to record changes between tissues and stages. Furthermore, somatic TE transposition may be limited to a few insertions in each cell making these hard to detect in healthy cells without the clonal amplification seen in cancer (Goodier, 2014). Although it is currently unknown just how fluid our somatic genomes are, recent technological advances have revealed mosaicisms of copy number variations within and between tissues (Abyzov et al., 2012; O'Huallachain et al., 2012) as well as extensive genetic heterogeneity in tumor cells (Aktipis and Nesse, 2013). As such, the 
notion of the mammalian somatic genome as a stable and inert code is questioned, and one cannot entirely rule out that certain somatic mutational profiles are adaptive for the organism as a whole.

It is evident that unleashed TE activity (e.g., caused by global aberration of genomic methylation) in neoplasm may induce further genetic changes accelerating cancer progression. However, the existence of stress-inducible TEs suggests that environmental stimuli alone may trigger the formation of genetic changes through TE activity at all stages of cancers, including the very onset. Similarly, it has recently been suggested that stress-induced TE activity may alter the neuronal genotypes in the human hippocampus, and that this could potentially be linked to neuronal disorders caused by severe stress (Hunter et al., 2013). Importantly, we do not advocate the view that TE activity underlies all human cancers. Lee and colleagues only found somatic TE activity in epithelial cancers (Lee et al., 2012), and TE involvement in cancers may be restricted to such plastic and reprogrammable tissues (Carreira et al., 2014).

The presented notion has several implications as different cellular stresses may elicit different TE responses in different cell types. Subsequently, different TE responses may underlie different cancers. This prompts for investigations not only into the genetic variability between cells in terms of TEs but also into the TE responses induced by cellular stress types. The advent of single-cell sequencing strategies (Evrony et al., 2012; Macaulay and Voet, 2014) combined with high-throughput sequencing technology makes it possible to test the above view and elucidate the involvement of TEs in cancer onset and progression. For example, exposing cell cultures to different external stresses should result in different TE responses, either in terms of TEs being activated or in the genomic regions targeted by their insertions. Similarly, careful examination of cancer types for which different stages of cancer progression is available should reveal fixed TE insertions present in the earliest stages. Hence, a combination of sequencing of stressed cells in vitro and of cancer cells in vivo could provide a rarely established link between the external environment and cancer genotypes in non-heritable cancers.

\section{ACKNOWLEDGMENT}

This work was supported by the Danish National Advanced Technology Foundation.

\section{REFERENCES}

Abyzov, A., Mariani, J., Palejev, D., Zhang, Y., Haney, M. S., Tomasini, L., et al. (2012). Somatic copy number mosaicism in human skin revealed by induced pluripotent stem cells. Nature 492, 438-442. doi: 10.1038/nature11629

Ahlquist, P. (2002). RNA-dependent RNA polymerases, viruses, and RNA silencing. Science 296, 1270-1273. doi: 10.1126/science. 1069132

Aktipis, C. A., and Nesse, R. M. (2013). Evolutionary foundations for cancer biology. Evol. Appl. 6, 144-159. doi: 10.1111/eva.12034

Allen, T. A., Von Kaenel, S., Goodrich, J. A., and Kugel, J. F. (2004). The SINEencoded mouse B2 RNA represses mRNA transcription in response to heat shock. Nat. Struct. Mol. Biol. 11, 816-821. doi: 10.1038/nsmb813

Aminetzach, Y. T., Macpherson, J. M., and Petrov, D. A. (2005). Pesticide resistance via transposition-mediated adaptive gene truncation in Drosophila. Science 309, 764-767. doi: 10.1126/science.1112699

Aravin, A. A., Sachidanandam, R., Girard, A., Fejes-Toth, K., and Hannon, G. J. (2007). Developmentally regulated piRNA clusters implicate MILI in transposon control. Science 316, 744-747. doi: 10.1126/science.1142612
Arkhipova, I., and Meselson, M. (2000). Transposable elements in sexual and ancient asexual taxa. Proc. Natl. Acad. Sci. U.S.A. 97, 14473-14477. doi: 10.1073/pnas.97.26.14473

Arnault, C., and Dufournel, I. (1994). Genome and stresses: reactions against aggressions, behavior of transposable elements. Genetica 93, 149-160. doi: 10.1007/BF01435247

Asch, H. L., Eliacin, E., Fanning, T. G., Connolly, J. L., Bratthauer, G., and Asch, B. B. (1996). Comparative expression of the LINE-1 p40 protein in human breast carcinomas and normal breast tissues. Oncol. Res. 8, 239-247.

Baillie, J. K., Barnett, M. W., Upton, K. R., Gerhardt, D. J., Richmond, T. A., De Sapio, F., et al. (2011). Somatic retrotransposition alters the genetic landscape of the human brain. Nature 479, 534-537. doi: 10.1038/nature10531

Batzer, M. A., and Deininger, P. L. (2002). Alu repeats and human genomic diversity. Nat. Rev. Genet. 3, 370-379. doi: 10.1038/nrg798

Bazak, L., Haviv, A., Barak, M., Jacob-Hirsch, J., Deng, P., Zhang, R., et al. (2014). A-to-I RNA editing occurs at over a hundred million genomic sites, located in a majority of human genes. Genome Res. 24, 365-376. doi: 10.1101/gr.1647 49.113

Bejerano, G., Lowe, C. B., Ahituv, N., King, B., Siepel, A., Salama, S. R., et al. (2006). A distal enhancer and an ultraconserved exon are derived from a novel retroposon. Nature 441, 87-90. doi: 10.1038/nature04696

Belancio, V. P., Roy-Engel, A. M., Pochampally, R. R., and Deininger, P. (2010). Somatic expression of LINE-1 elements in human tissues. Nucleic Acids Res. 38, 3909-3922. doi: 10.1093/nar/gkq132

Belshaw, R., Dawson, A. L., Woolven-Allen, J., Redding, J., Burt, A., and Tristem, M. (2005). Genomewide screening reveals high levels of insertional polymorphism in the human endogenous retrovirus family HERV-K(HML2): implications for present-day activity. J. Virol. 79, 12507-12514. doi: 10.1128/JVI.79.19.1250712514.2005

Bennett, E. A., Coleman, L. E., Tsui, C., Pittard, W. S., and Devine, S. E. (2004). Natural genetic variation caused by transposable elements in humans. Genetics 168, 933-951. doi: 10.1534/genetics.104.031757

Bergman, A., and Siegal, M. L. (2003). Evolutionary capacitance as a general feature of complex gene networks. Nature 424, 549-552. doi: 10.1038/nature 01765

Boissinot, S., Davis, J., Entezam, A., Petrov, D., and Furano, A. V. (2006). Fitness cost of LINE-1 (L1) activity in humans. Proc. Natl. Acad. Sci. U.S.A. 103, 9590-9594. doi: 10.1073/pnas.0603334103

Boissinot, S., Entezam, A., and Furano, A. V. (2001). Selection against deleterious LINE-1-containing loci in the human lineage. Mol. Biol. Evol. 18, 926-935. doi: 10.1093/oxfordjournals.molbev.a003893

Boller, K., Schonfeld, K., Lischer, S., Fischer, N., Hoffmann, A., Kurth, R., et al. (2008). Human endogenous retrovirus HERV-K113 is capable of producing intact viral particles. J. Gen. Virol. 89, 567-572. doi: 10.1099/vir.0.83534-0

Bowen, N. J., Jordan, I. K., Epstein, J. A., Wood, V., and Levin, H. L. (2003). Retrotransposons and their recognition of pol II promoters: a comprehensive survey of the transposable elements from the complete genome sequence of Schizosaccharomyces pombe. Genome Res. 13, 1984-1997. doi: 10.1101/gr. 1191603

Brouha, B., Schustak, J., Badge, R. M., Lutz-Prigge, S., Farley, A. H., Moran, J. V., etal. (2003). Hot Lls account for the bulk of retrotransposition in the human population. Proc. Natl. Acad. Sci. U.S.A. 100, 5280-5285. doi: 10.1073/pnas.0831042100

Capy, P., Gasperi, G., Biemont, C., and Bazin, C. (2000). Stress and transposable elements: co-evolution or useful parasites? Heredity (Edinb) 85(Pt 2), 101-106. doi: 10.1046/j.1365-2540.2000.00751.x

Carmell, M. A., Girard, A., Van De Kant, H. J., Bourc'his, D., Bestor, T. H., De Rooij, D. G., et al. (2007). MIWI2 is essential for spermatogenesis and repression of transposons in the mouse male germline. Dev. Cell 12, 503-514. doi: 10.1016/j.devcel.2007.03.001

Carreira, P. E., Richardson, S. R., and Faulkner, G. J. (2014). L1 retrotransposons, cancer stem cells and oncogenesis. FEBS J. 281, 63-73. doi: 10.1111/febs. 12601

Chen, J. M., Stenson, P. D., Cooper, D. N., and Ferec, C. (2005). A systematic analysis of LINE-1 endonuclease-dependent retrotranspositional events causing human genetic disease. Hum. Genet. 117, 411-427. doi: 10.1007/s00439-005-1321-0

Chen, L. L., Decerbo, J. N., and Carmichael, G. G. (2008). Alu element-mediated gene silencing. EMBO J. 27, 1694-1705. doi: 10.1038/Emboj.2008.94 
Chuong, E. B. (2013). Retroviruses facilitate the rapid evolution of the mammalian placenta. Bioessays 35, 853-861. doi: 10.1002/bies.201300059

Chuong, E. B., Rumi, M. A., Soares, M. J., and Baker, J. C. (2013). Endogenous retroviruses function as species-specific enhancer elements in the placenta. Nat. Genet. 45, 325-329. doi: 10.1038/ng.2553

Cordaux, R., and Batzer, M. A. (2009). The impact of retrotransposons on human genome evolution. Nat. Rev. Genet. 10, 691-703. doi: 10.1038/nrg2640

Daniels, G. R., and Deininger, P. L. (1985). Repeat sequence families derived from mammalian tRNA genes. Nature 317, 819-822. doi: 10.1038/317 $819 \mathrm{a} 0$

Darboux, I., Charles, J. F., Pauchet, Y., Warot, S., and Pauron, D. (2007). Transposonmediated resistance to Bacillus sphaericus in a field-evolved population of Culex pipiens (Diptera: Culicidae). Cell. Microbiol. 9, 2022-2029. doi: 10.1111/j.14625822.2007.00934.x

Daskalos, A., Nikolaidis, G., Xinarianos, G., Savvari, P., Cassidy, A., Zakopoulou, R., et al. (2009). Hypomethylation of retrotransposable elements correlates with genomic instability in non-small cell lung cancer. Int. J. Cancer 124, 81-87. doi: 10.1002/ijc. 23849

Deininger, P. L., Moran, J. V., Batzer, M. A., and Kazazian, H. H. Jr. (2003). Mobile elements and mammalian genome evolution. Curr. Opin. Genet. Dev. 13, 651658. doi: 10.1016/j.gde.2003.10.013

de Koning, A. P., Gu, W., Castoe, T. A., Batzer, M. A., and Pollock, D. D. (2011) Repetitive elements may comprise over two-thirds of the human genome. PLoS Genet. 7:e1002384. doi: 10.1371/journal.pgen.1002384

Dewannieux, M., Esnault, C., and Heidmann, T. (2003). LINE-mediated retrotransposition of marked Alu sequences. Nat. Genet. 35, 41-48. doi: 10.1038/ ng1223

Dunn, C. A., Medstrand, P., and Mager, D. L. (2003). An endogenous retroviral long terminal repeat is the dominant promoter for human $\beta$ 1,3-galactosyltransferase 5 in the colon. Proc. Natl. Acad. Sci. U.S.A. 100, 12841-12846. doi: 10.1073/pnas.2134464100

Dunn, C. A., Romanish, M. T., Gutierrez, L. E., Van De Lagemaat, L. N., and Mager, D. L. (2006). Transcription of two human genes from a bidirectional endogenous retrovirus promoter. Gene 366, 335-342. doi: 10.1016/j.gene.2005 09.003

Ebina, H., and Levin, H. L. (2007). Stress management: how cells take control of their transposons. Mol. Cell 27, 180-181. doi: 10.1016/j.molcel.2007.07.004

Esnault, C., Maestre, J., and Heidmann, T. (2000). Human LINE retrotransposons generate processed pseudogenes. Nat. Genet. 24, 363-367. doi: 10.1038/ 74184

Evrony, G. D., Cai, X., Lee, E., Hills, L. B., Elhosary, P. C., Lehmann, H. S., et al. (2012). Single-neuron sequencing analysis of L1 retrotransposition and somatic mutation in the human brain. Cell 151, 483-496. doi: 10.1016/j.cell.2012.09.035

Faulkner, G. J., Kimura, Y., Daub, C. O., Wani, S., Plessy, C., Irvine, K. M., et al. (2009). The regulated retrotransposon transcriptome of mammalian cells. Nat Genet. 41, 563-571. doi: 10.1038/ng.368

Feng, G., Leem, Y. E., and Levin, H. L. (2012). Transposon integration enhances expression of stress response genes. Nucleic Acids Res. 41, 775-789. doi: 10.1093/nar/gks1185

Frost, R. J., Hamra, F. K., Richardson, J. A., Qi, X., Bassel-Duby, R., and Olson, E. N. (2010). MOV10L1 is necessary for protection of spermatocytes against retrotransposons by Piwi-interacting RNAs. Proc. Natl. Acad. Sci. U.S.A. 107, 11847-11852. doi: 10.1073/pnas. 1007158107

Gai, X., and Voytas, D. F. (1998). A single amino acid change in the yeast retrotransposon Ty5 abolishes targeting to silent chromatin. Mol. Cell 1, 1051-1055. doi: 10.1016/S1097-2765(00)80105-7

Garcia Guerreiro, M. P. (2012). What makes transposable elements move in the Drosophila genome? Heredity (Edinb) 108, 461-468. doi: 10.1038/hdy.2011.89

Gardner, M. J., Hall, N., Fung, E., White, O., Berriman, M., Hyman, R. W., et al. (2002). Genome sequence of the human malaria parasite Plasmodium falciparum. Nature 419, 498-511. doi: 10.1038/nature01097

Goodier, J. L. (2014). Retrotransposition in tumors and brains. Mob. DNA 5, 11. doi: $10.1186 / 1759-8753-5-11$

Goodier, J. L., Cheung, L. E., and Kazazian, H. H. Jr. (2012). MOV10 RNA helicase is a potent inhibitor of retrotransposition in cells. PLoS Genet. 8:e1002941, doi: $10.1093 / \mathrm{nar} / \mathrm{gks} 1185$

Goodier, J. L., Zhang, L., Vetter, M. R., and Kazazian, H. H. Jr. (2007). LINE-1 ORF1 protein localizes in stress granules with other RNA-binding proteins, including components of RNA interference RNA-induced silencing complex. Mol. Cell. Biol. 27, 6469-6483. doi: 10.1128/MCB.00332-07

Grandbastien, M. A., Lucas, H., Morel, J. B., Mhiri, C., Vernhettes, S., and Casacuberta, J. M. (1997). The expression of the tobacco Tntl retrotransposon is linked to plant defense responses. Genetica 100, 241-252. doi: 10.1023/A:1018302 216927

Gualtieri, A., Andreola, F., Sciamanna, I., Sinibaldi-Vallebona, P., Serafino, A., and Spadafora, C. (2013). Increased expression and copy number amplification of LINE-1 and SINE B1 retrotransposable elements in murine mammary carcinoma progression. Oncotarget 4, 1882-1893.

Haig, D. (2012). Retroviruses and the placenta. Curr. Biol. 22, R609-R613. doi: 10.1016/j.cub.2012.06.002

Han, J. S., Szak, S. T., and Boeke, J. D. (2004). Transcriptional disruption by the L1 retrotransposon and implications for mammalian transcriptomes. Nature 429, 268-274. doi: 10.1038/nature02536

Han, K., Sen, S. K., Wang, J., Callinan, P. A., Lee, J., Cordaux, R., et al. (2005). Genomic rearrangements by LINE-1 insertion-mediated deletion in the human and chimpanzee lineages. Nucleic Acids Res. 33, 4040-4052. doi: 10.1093/nar/gki718

Hancks, D. C., and Kazazian, H. H. Jr. (2012). Active human retrotransposons: variation and disease. Curr. Opin. Genet. Dev. 22, 191-203. doi 10.1016/j.gde.2012.02.006

Hauptmann, S., and Schmitt, W. D. (2006). Transposable elements - is there a link between evolution and cancer? Med. Hypotheses 66, 580-591. doi: 10.1016/j.mehy.2005.08.051

Hedges, D. J., Callinan, P. A., Cordaux, R., Xing, J., Barnes, E., and Batzer, M. A. (2004). Differential Alu mobilization and polymorphism among the human and chimpanzee lineages. Genome Res. 14, 1068-1075. doi: 10.1101/gr.25 30404

Hermisson, J., and Wagner, G. P. (2004). The population genetic theory of hidden variation and genetic robustness. Genetics 168, 2271-2284. doi: 10.1534/genetics. 104.029173

Howard, G., Eiges, R., Gaudet, F., Jaenisch, R., and Eden, A. (2008). Activation and transposition of endogenous retroviral elements in hypomethylation induced tumors in mice. Oncogene 27, 404-408. doi: 10.1038/sj.onc.1210631

Hughes, J. F., and Coffin, J. M. (2001). Evidence for genomic rearrangements mediated by human endogenous retroviruses during primate evolution. Nat. Genet. 29, 487-489. doi: 10.1038/ng775

Hunter, R. G., Mcewen, B. S., and Pfaff, D. W. (2013). Environmental stress and transposon transcription in the mammalian brian. Mob. Genet. Elements 3, e24555. doi: 10.4161/mge.24555

Hunter, R. G., Murakami, G., Dewell, S., Seligsohn, M., Baker, M. E., Datson, N. A., et al. (2012). Acute stress and hippocampal histone H3 lysine 9 trimethylation, a retrotransposon silencing response. Proc. Natl. Acad. Sci. U.S.A. 109, $17657-$ 17662. doi: 10.1073/pnas.1215810109

Ishizaka, Y., Okudaira, N., Tamura, M., Iijima, K., Shimura, M., Goto, M., et al. (2012). Modes of retrotransposition of long interspersed element-1 by environmental factors. Front. Microbiol 3:191. doi: 10.3389/fmicb.2012. 00191

Kanazawa, A., Liu, B., Kong, F., Arase, S., and Abe, J. (2009). Adaptive evolution involving gene duplication and insertion of a novel Tyl/copia-like retrotransposon in soybean. J. Mol. Evol. 69, 164-175. doi: 10.1007/s00239-009-9262-1

Kazazian, H. H. Jr. (2004). Mobile elements: drivers of genome evolution. Science 303, 1626-1632. doi: 10.1126/science.1089670

Keren, H., Lev-Maor, G., and Ast, G. (2010). Alternative splicing and evolution: diversification, exon definition and function. Nat. Rev. Genet. 11, 345-355. doi: $10.1038 / \operatorname{nrg} 2776$

Kuramochi-Miyagawa, S., Watanabe, T., Gotoh, K., Totoki, Y., Toyoda, A., Ikawa, M., et al. (2008). DNA methylation of retrotransposon genes is regulated by Piwi family members MILI and MIWI2 in murine fetal testes. Genes Dev. 22, 908-917. doi: $10.1101 /$ gad. 1640708

Kuwabara, T., Hsieh, J., Muotri, A., Yeo, G., Warashina, M., Lie, D. C., et al. (2009). Wnt-mediated activation of NeuroD1 and retro-elements during adult neurogenesis. Nat. Neurosci. 12, 1097-1105. doi: 10.1038/nn.2360

Lamprecht, B., Walter, K., Kreher, S., Kumar, R., Hummel, M., Lenze, D., et al. (2010). Derepression of an endogenous long terminal repeat activates the CSF1R proto-oncogene in human lymphoma. Nat. Med. 16, 571-579. doi: 10.1038/ nm.2129 
Lander, E. S., Linton, L. M., Birren, B., Nusbaum, C., Zody, M. C., Baldwin, J., et al. (2001). Initial sequencing and analysis of the human genome. Nature 409, 860-921. doi: 10.1038/35057062

Lee, E., Iskow, R., Yang, L., Gokcumen, O., Haseley, P., Luquette, L. J., et al. (2012). Landscape of somatic retrotransposition in human cancers. Science 337, 967-971. doi: $10.1126 /$ science. 1222077

Lee, J., Cordaux, R., Han, K., Wang, J., Hedges, D. J., Liang, P., et al. (2007) Different evolutionary fates of recently integrated human and chimpanzee LINE1 retrotransposons. Gene 390, 18-27. doi: 10.1016/j.gene.2006.08.029

Leem, Y. E., Ripmaster, T. L., Kelly, F. D., Ebina, H., Heincelman, M. E., Zhang, K., et al. (2008). Retrotransposon Tf1 is targeted to Pol II promoters by transcription activators. Mol. Cell 30, 98-107. doi: 10.1016/j.molcel.2008.02.016

Levin, H. L., and Moran, J. V. (2011). Dynamic interactions between transposable elements and their hosts. Nat. Rev. Genet. 12, 615-627. doi: 10.1038/ nrg3030

Levy, S., Sutton, G., Ng, P. C., Feuk, L., Halpern, A. L., Walenz, B. P., et al. (2007). The diploid genome sequence of an individual human. PLoS Biol. 5:e254. doi 10.1371/journal.pbio.0050254

Li, J., Kannan, M., Trivett, A. L., Liao, H., Wu, X., Akagi, K., et al. (2014). An antisense promoter in mouse $\mathrm{L} 1$ retrotransposon open reading frame-1 initiates expression of diverse fusion transcripts and limits retrotransposition. Nucleic Acids Res. 42, 4546-4562. doi: 10.1093/nar/gku091

Lin, L., Shen, S., Tye, A., Cai, J. J., Jiang, P., Davidson, B. L., et al. (2008). Diverse splicing patterns of exonized Alu elements in human tissues. PLoS Genet. 4:e1000225 doi: 10.1371/journal.pgen.1000225

Liu, W.-M., Chu, W.-M., Choudary, P. V., and Schmid, C. W. (1995). Cell stress and translational inhibitors transiently increase the abundance of mammalian SINE transcripts. Nucleic Acids Res. 23, 1758-1765. doi: 10.1093/nar/23. 10.1758

Lunyak, V. V., Prefontaine, G. G., Nunez, E., Cramer, T., Ju, B.-G., Ohgi, K. A., et al. (2007). Developmentally regulated activation of a SINE B2 repeat as a domain boundary in organogenesis. Science 317, 248-251. doi: 10.1126/science.11 40871

Macaulay, I. C., and Voet, T. (2014). Single cell genomics: advances and future perspectives. PLoS Genet. 10:e1004126. doi: 10.1371/journal.pgen.1004126

Mariner, P. D., Walters, R. D., Espinoza, C. A., Drullinger, L. F., Wagner, S. D., Kugel, J. F., et al. (2008). Human Alu RNA is a modular transacting repressor of mRNA transcription during heat shock. Mol. Cell 29, 499-509. doi: 10.1016/j.molcel.2007.12.013

Masel, J., and Trotter, M. V. (2010). Robustness and evolvability. Trends Genet. 26, 406-414. doi: 10.1016/j.tig.2010.06.002

McClintock, B. (1984). The significance of responses of the genome to challenge. Science 226, 792-801. doi: 10.1126/science.15739260

Mhiri, C., Morel, J. B., Vernhettes, S., Casacuberta, J. M., Lucas, H., and Grandbastien, M. A. (1997). The promoter of the tobacco Tntl retrotransposon is induced by wounding and by abiotic stress. Plant Mol. Biol. 33, 257-266. doi 10.1023/A:1005727132202

Morales, J. F., Snow, E. T., and Murnane, J. P. (2003). Environmental factors affecting transcription of the human L1 retrotransposon. II. Stressors. Mutagenesis 18, 151-158. doi: 10.1093/mutage/18.2.151

Mourier, T. (2005). Reverse transcription in genome evolution. Cytogenet. Genome Res. 110, 56-62. doi: 10.1159/000084938

Mourier, T. (2011). Retrotransposon-centered analysis of piRNA targeting shows a shift from active to passive retrotransposon transcription in developing mouse testes. BMC Genomics 12, 440. doi: 10.1186/1471-2164-12-440

Muotri, A. R., Chu, V. T., Marchetto, M. C., Deng, W., Moran, J. V., and Gage, F. H. (2005). Somatic mosaicism in neuronal precursor cells mediated by L1 retrotransposition. Nature 435, 903-910. doi: 10.1038/nature 03663

O’Huallachain, M., Karczewski, K. J., Weissman, S. M., Urban, A. E., and Snyder, M. P. (2012). Extensive genetic variation in somatic human tissues. Proc. Natl. Acad. Sci. U.S.A. 109, 18018-18023. doi: 10.1073/pnas.1213736109

Ostertag, E. M., Goodier, J. L., Zhang, Y., and Kazazian, H. H. Jr. (2003). SVA elements are nonautonomous retrotransposons that cause disease in humans. Am. J. Hum. Genet. 73, 1444-1451. doi: 10.1086/380207

Ostertag, E. M., and Kazazian, H. H. Jr. (2001). Biology of mammalian L1 retrotransposons. Annu. Rev. Genet. 35, 501-538. doi: 10.1146/annurev.genet.35.102401.091032
Pace, J. K. II, and Feschotte, C. (2007). The evolutionary history of human DNA transposons: evidence for intense activity in the primate lineage. Genome Res. 17, 422-432. doi: 10.1101/gr.5826307

Pavicic, W., Joensuu, E. I., Nieminen, T., and Peltomaki, P. (2012). LINE-1 hypomethylation in familial and sporadic cancer. J. Mol. Med. (Berl.) 90, 827-835. doi: 10.1007/s00109-011-0854-Z

Paz-Yaacov, N., Levanon, E. Y., Nevo, E., Kinar, Y., Harmelin, A., JacobHirsch, J., et al. (2010). Adenosine-to-inosine RNA editing shapes transcriptome diversity in primates. Proc. Natl. Acad. Sci. U.S.A. 107, 12174-12179. doi: 10.1073/pnas.1006183107

Penzkofer, T., Dandekar, T., and Zemojtel, T. (2005). L1Base: from functional annotation to prediction of active LINE-1 elements. Nucleic Acids Res. 33, D498-D500. doi: 10.1093/nar/gki044

Pickeral, O. K., Makalowski, W., Boguski, M. S., and Boeke, J. D. (2000). Frequent human genomic DNA transduction driven by LINE-1 retrotransposition. Genome Res. 10, 411-415. doi: 10.1101/gr.10.4.411

Ponicsan, S. L., Kugel, J. F., and Goodrich, J. A. (2010). Genomic gems: SINE RNAs regulate mRNA production. Curr. Opin. Genet. Dev. 20, 149-155. doi: 10.1016/j.gde.2010.01.004

Poole, A. M., Phillips, M. J., and Penny, D. (2003). Prokaryote and eukaryote evolvability. Biosystems 69, 163-185. doi: 10.1016/S0303-2647(02) 00131-4

Richter, K., Haslbeck, M., and Buchner, J. (2010). The heat shock response: life on the verge of death. Mol. Cell 40, 253-266. doi: 10.1016/j.molcel.2010. 10.006

Roman-Gomez, J., Jimenez-Velasco, A., Agirre, X., Cervantes, F., Sanchez, J., Garate, L., et al. (2005). Promoter hypomethylation of the LINE-1 retrotransposable elements activates sense/antisense transcription and marks the progression of chronic myeloid leukemia. Oncogene 24, 7213-7223. doi: 10.1038/sj.onc.12 08866

Romanish, M. T., Cohen, C. J., and Mager, D. L. (2010). Potential mechanisms of endogenous retroviral-mediated genomic instability in human cancer. Semin. Cancer Biol. 20, 246-253. doi: 10.1016/j.semcancer.2010.05.005

Rutherford, S. L., and Lindquist, S. (1998). Hsp90 as a capacitor for morphological evolution. Nature 396, 336-342. doi: 10.1038/24550

Schumann, G. G. (2007). APOBEC3 proteins: major players in intracellular defence against LINE-1-mediated retrotransposition. Biochem. Soc. Trans. 35, 637-642. doi: 10.1042/BST0350637

Sehgal, A., Lee, C. Y., and Espenshade, P. J. (2007). SREBP controls oxygendependent mobilization of retrotransposons in fission yeast. PLoS Genet. 3:e131. doi: 10.1371/journal.pgen.003013

Sen, S. K., Han, K., Wang, J., Lee, J., Wang, H., Callinan, P. A., et al. (2006). Human genomic deletions mediated by recombination between Alu elements. Am. J. Hum. Genet. 79, 41-53. doi: 10.1086/504600

Servant, G., Pinson, B., Tchalikian-Cosson, A., Coulpier, F., Lemoine, S., Pennetier, C., et al. (2012). Tye7 regulates yeast Tyl retrotransposon sense and antisense transcription in response to adenylic nucleotides stress. Nucleic Acids Res. 40, 5271-5282. doi: 10.1093/nar/gks166

Slotkin, R. K., and Martienssen, R. (2007). Transposable elements and the epigenetic regulation of the genome. Nat. Rev. Genet. 8, 272-285. doi: 10.1038/nrg2072

Specchia, V., Piacentini, L., Tritto, P., Fanti, L., D’Alessandro, R., Palumbo, G., et al. (2010). Hsp90 prevents phenotypic variation by suppressing the mutagenic activity of transposons. Nature 463, 662-665. doi: 10.1038/nature 08739

Stetson, D. B., Ko, J. S., Heidmann, T., and Medzhitov, R. (2008). Trex1 prevents cell-intrinsic initiation of autoimmunity. Cell 134, 587-598. doi: 10.1016/j.cell.2008.06.032

Stewart, C., Kural, D., Stromberg, M. P., Walker, J. A., Konkel, M. K., Stutz, A. M., et al. (2011). A comprehensive map of mobile element insertion polymorphisms in humans. PLoS Genet. 7:e1002236. doi: 10.1371/journal.pgen. 1002236

van de Lagemaat, L. N., Landry, J. R., Mager, D. L., and Medstrand, P. (2003) Transposable elements in mammals promote regulatory variation and diversification of genes with specialized functions. Trends Genet. 19, 530-536. doi: 10.1016/j.tig.2003.08.004

Wang, H., Xing, J., Grover, D., Hedges, D. J., Han, K., Walker, J. A., et al. (2005). SVA elements: a hominid-specific retroposon family. J. Mol. Biol. 354, 994-1007. doi: 10.1016/j.jmb.2005.09.085 
Wang, J., Song, L., Gonder, M. K., Azrak, S., Ray, D. A., Batzer, M. A., et al. (2006). Whole genome computational comparative genomics: a fruitful approach for ascertaining Alu insertion polymorphisms. Gene 365, 11-20. doi: 10.1016/j.gene.2005.09.031

Wang, J., Wang, W., Li, R., Li, Y., Tian, G., Goodman, L., et al. (2008). The diploid genome sequence of an Asian individual. Nature 456, 60-65. doi: 10.1038/nature07484

Wilson, A. S., Power, B. E., and Molloy, P. L. (2007). DNA hypomethylation and human diseases. Biochim. Biophys. Acta 1775, 138-162.

Wimmer, K., Callens, T., Wernstedt, A., and Messiaen, L. (2011). The NF1 gene contains hotspots for $\mathrm{L} 1$ endonuclease-dependent de novo insertion. PLoS Genet. 7:e1002371. doi: 10.1371/journal.pgen.1002236

Wolff, E. M., Byun, H. M., Han, H. F., Sharma, S., Nichols, P. W., Siegmund, K. D., et al. (2010). Hypomethylation of a LINE-1 promoter activates an alternate transcript of the MET oncogene in bladders with cancer. PLoS Genet. 6:e1000917. doi: 10.1371/journal.pgen.1000917

Xiang, S., Liu, Z., Zhang, B., Zhou, J., Zhu, B. D., Ji, J., et al. (2010). Methylation status of individual CpG sites within Alu elements in the human genome and Alu hypomethylation in gastric carcinomas. BMC Cancer 10:44. doi: 10.1186/14712407-10-44

Xie, M., Hong, C., Zhang, B., Lowdon, R. F., Xing, X., Li, D., et al. (2013). DNA hypomethylation within specific transposable element families associates with tissue-specific enhancer landscape. Nat. Genet. 45, 836-841. doi: 10.1038/ng.2649
Yang, N., and Kazazian, H. H. (2006). L1 retrotransposition is suppressed by endogenously encoded small interfering RNAs in human cultured cells. Nat. Struct. Mol. Biol. 13, 763-771. doi: 10.1038/nsmb1141

Zhang, Z., Harrison, P. M., Liu, Y., and Gerstein, M. (2003). Millions of years of evolution preserved: a comprehensive catalog of the processed pseudogenes in the human genome. Genome Res. 13, 2541-2558. doi: 10.1101/gr.1429003

Conflict of Interest Statement: The authors declare that the research was conducted in the absence of any commercial or financial relationships that could be construed as a potential conflict of interest.

Received: 07 March 2014; accepted: 11 May 2014; published online: 30 May 2014.

Citation: Mourier T, Nielsen LP, Hansen AJ and Willerslev E (2014) Transposable elements in cancer as a by-product of stress-induced evolvability. Front. Genet. 5:156. doi: 10.3389/fgene.2014.00156

This article was submitted to Evolutionary and Population Genetics, a section of the journal Frontiers in Genetics.

Copyright (C) 2014 Mourier, Nielsen, Hansen and Willerslev. This is an open-access article distributed under the terms of the Creative Commons Attribution License (CC BY). The use, distribution or reproduction in other forums is permitted, provided the original author(s) or licensor are credited and that the original publication in this journal is cited, in accordance with accepted academic practice. No use, distribution or reproduction is permitted which does not comply with these terms. 